\title{
Application of the LEPS technique for Quantitative Precipitation Forecasting (QPF) in Southern Italy: a preliminary study
}

\author{
S. Federico ${ }^{1,2}$, E. Avolio ${ }^{1,3}$, C. Bellecci ${ }^{1,3}$, M. Colacino ${ }^{2}$, and R. L. Walko ${ }^{4}$ \\ ${ }^{1}$ CRATI Scrl, c/o University of Calabria, 87036 Rende (CS), Italy \\ ${ }^{2}$ CNR-ISAC, via del Fosso del Cavaliere, 100, 00133 Rome, Italy \\ ${ }^{3}$ University of Rome "Tor Vergata" Dept. STFE, via del Politecnico, 1, 00133 Rome, Italy \\ ${ }^{4}$ Pratt School of Engineering, Duke University, Durham, NC, USA
}

Received: 7 June 2005 - Revised: 30 January 2006 - Accepted: 30 January 2006 - Published: 24 March 2006

\begin{abstract}
This paper reports preliminary results for a Limited area model Ensemble Prediction System (LEPS), based on RAMS (Regional Atmospheric Modelling System), for eight case studies of moderate-intense precipitation over Calabria, the southernmost tip of the Italian peninsula. LEPS aims to transfer the benefits of a probabilistic forecast from global to regional scales in countries where local orographic forcing is a key factor to force convection.

To accomplish this task and to limit computational time in an operational implementation of LEPS, we perform a cluster analysis of ECMWF-EPS runs. Starting from the 51 members that form the ECMWF-EPS we generate five clusters. For each cluster a representative member is selected and used to provide initial and dynamic boundary conditions

To further explore the impact of local physiographic features on QPF (Quantitative Precipitation Forecasting), LEPS results are also compared with a 6-km horizontal resolution deterministic forecast. Due to local and mesoscale forcing, the high resolution forecast (Hi-Res) has better performance compared to the ensemble mean for rainfall thresholds larger than $10 \mathrm{~mm}$ but it tends to overestimate precipitation for lower amounts. This yields larger false alarms that have a detrimental effect on objective scores for lower thresholds.

To exploit the advantages of a probabilistic forecast compared to a deterministic one, the relation between the ECMWF-EPS $700 \mathrm{hPa}$ geopotential height spread and LEPS performance is analyzed. Results are promising even if additional studies are required.
\end{abstract} to RAMS, whose integrations generate LEPS. RAMS runs have $12-\mathrm{km}$ horizontal resolution.

To analyze the impact of enhanced horizontal resolution on quantitative precipitation forecasts, LEPS forecasts are compared to a full Brute Force (BF) ensemble. This ensemble is based on RAMS, has $36 \mathrm{~km}$ horizontal resolution and is generated by 51 members, nested in each ECMWF-EPS member.

LEPS and BF results are compared subjectively and by objective scores. Subjective analysis is based on precipitation and probability maps of case studies whereas objective analysis is made by deterministic and probabilistic scores. Scores and maps are calculated by comparing ensemble precipitation forecasts against reports from the Calabria regional raingauge network.

Results show that LEPS provided better rainfall predictions than BF for all case studies selected. This strongly suggests the importance of the enhanced horizontal resolution, compared to ensemble population, for Calabria for these cases.

Correspondence to: S. Federico

(s.federico@crati.it)

\section{Introduction}

Numerical Weather Prediction (NWP) model performance has noticeably improved in the last 15 years due to several reasons such as increased computer power, more detailed physical and numerical parameterizations, better processes formulation, and improvements in data assimilation. In addition, the increase of computer power has enabled the use of a General Circulation Model (GCM) Ensemble Prediction System (EPS) in several meteorological centers around the world (Molteni et al., 1996; Houtemaker et al. 1996; Toth and Kalnay, 1994).

Despite these improvements, forecasting of highly localized and severe events is still a challenging problem in many areas, particularly where orographic features and mesoscale structures cannot be properly represented by GCM-EPS because of its coarse horizontal resolution. At the time of this study, the ECMWF-EPS uses a T255L40 configuration that corresponds, for mid latitudes, to about 80 -km horizontal resolution. This resolution is not enough to represent orographic

Published by Copernicus GmbH on behalf of the European Geosciences Union and the American Geophysical Union. 


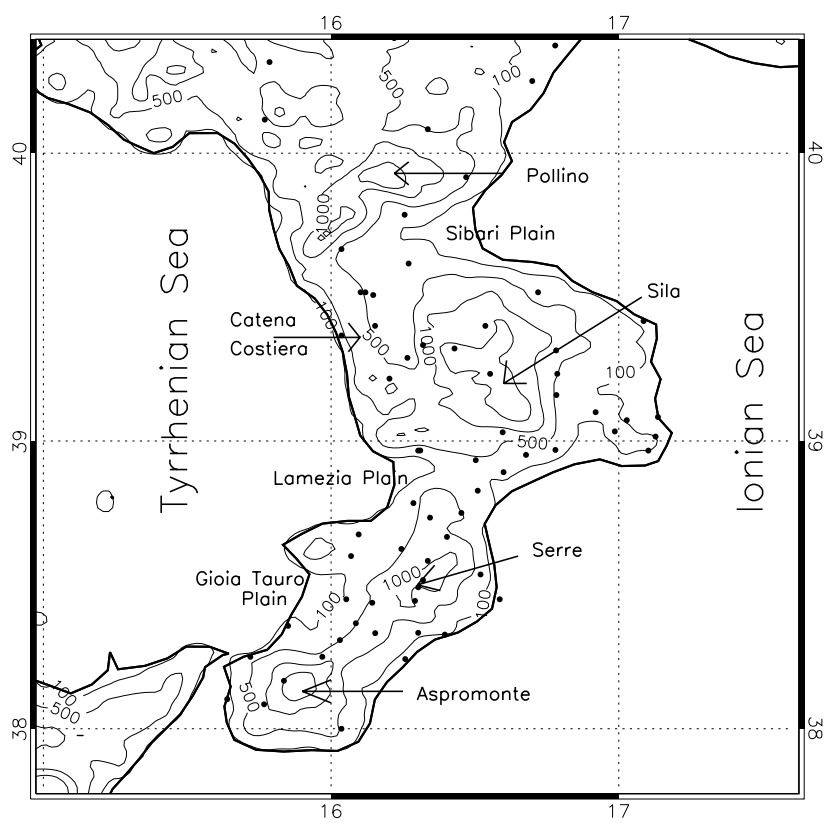

Fig. 1. Topography of Calabria averaged over $10 \mathrm{~km}^{2}$. Main features are also reported. Contours: $100 \mathrm{~m}, 500 \mathrm{~m}, 1000 \mathrm{~m}$ and $1400 \mathrm{~m}$. Dots show raingauge locations.

forcing and mesoscale structures of Calabria, the southernmost tip of the Italian boot, where this study is focused.

Calabria ranges from $37^{\circ} 55^{\prime}$ to $40^{\circ}$ North latitude and $15^{\circ} 30^{\prime}$ to $17^{\circ} 15^{\prime}$ East longitude (Fig. 1). The average width of the region is about $300 \mathrm{~km}$ north to south and $50 \mathrm{~km}$ west to east. The Apennines run North to South and are characterized by five main topographical features that reach 1.5$2.0 \mathrm{~km}$ in elevation: Pollino, Catena Costiera, Sila, Serre, and Aspromonte. The west coast of Calabria is bounded by the Tyrrhenian Sea. The east and south coasts are bounded by the Ionian Sea. Three main valleys are located near the sea: the Larnezia Plain and the Gioia Tauro Plain on the west, and the Sibari Plain on the east. Most of the region's agricultural and industrial sites are located in these valleys.

There are three main goals of EPS: (a) improve forecast skill by averaging unpredictable components, thereby reducing the impact of non-linear error growth; (b) evaluate forecast skill by relating it to the agreement among ensemble members; (c) provide an objective basis for casting the forecast in a probabilistic form.

In order to gain the advantages of EPS in a region like Calabria, the Limited area model Ensemble Prediction System (LEPS) approach is investigated for eight case studies. LEPS (Marsigli et al., 2001; Montani et al., 2003) aims to transport advantages of the ECMWF-EPS forecast at the mesoscaleregional scale by a limited area model to produce probabilistic forecasts. Nevertheless, currently-available computer resources are not sufficient to implement it in an operational framework. To reduce computing time, LEPS uses a cluster technique (Molteni et al., 2001). In our study a hierarchical cluster analysis is implemented and used to reduce the whole ECMWF-EPS to five clusters. For each of them, a representative member (RM) is selected to force the multiscale, multipurpose meteorological model RAMS (Regional Atmospheric Modelling System, Pielke et al., 1992; Cotton et al., 2003). Distance between ensemble members is computed by considering four meteorological variables on three pressure levels. These meteorological discriminating variables are zonal and meridional wind components $(\mathrm{u}, \mathrm{v})$, relative humidity (RH) and geopotential height (Z) for pressure levels $850 \mathrm{hPa}, 700 \mathrm{hPa}$ and $500 \mathrm{hPa}$. Variables and levels were selected by trial and error in order to transfer key synoptic scale features from cluster elements to representative members. This system is tested for eight storms that produced moderate-intense rainfall over Calabria.

The paper is organized as follows: Sect. 2 describes the methodology followed to generate clusters and RMs, Sect. 3 introduces model set-up, Sect. 4 presents the results along with the methodology followed to verify forecasts, and Sect. 5 gives conclusions.

\section{The cluster methodology and LEPS approach}

The methodology adopted to cluster the ECMWF-EPS members and to select the RM from each cluster was proposed by Molteni et al. (2001) and was adapted and used in Marsigli et al. (2001) and in Montani et al. (2003). In this study, we make minor changes to this methodology in order to better focus on the study area, which is the central Mediterranean basin, and to account for event duration. Our approach is as follows:

1. We begin with 51 clusters where each ECMWF-EPS member belongs to one cluster.

2. From $t_{0}+60 h$ to $t_{0}+84 h$, where $t_{0}$ is the initial simulation time, we compute the standardized anomalies of the discriminating variables $(\mathrm{u}, \mathrm{v}, \mathrm{RH}, \mathrm{Z})$ for three pressure levels $(850 \mathrm{hPa}, 700 \mathrm{hPa}, 500 \mathrm{hPa})$.

3. We calculate the space-averaged quadratic distances between all ECMWF-EPS members for each discriminating variable standardized anomaly and for each level. Distances are computed over a target area that, in our case, spans the central Mediterranean basin $\left(5^{\circ} \mathrm{E}-20^{\circ} \mathrm{E} ; 35^{\circ} \mathrm{N}-45^{\circ} \mathrm{N}\right)$.

4. Next, we calculate distances between different cluster elements, averaging standardized anomaly distances over all discriminating variables and levels. Distance between clusters is computed by averaging distances between all cluster members.

5. The two clusters having the minimum distance are grouped together. 


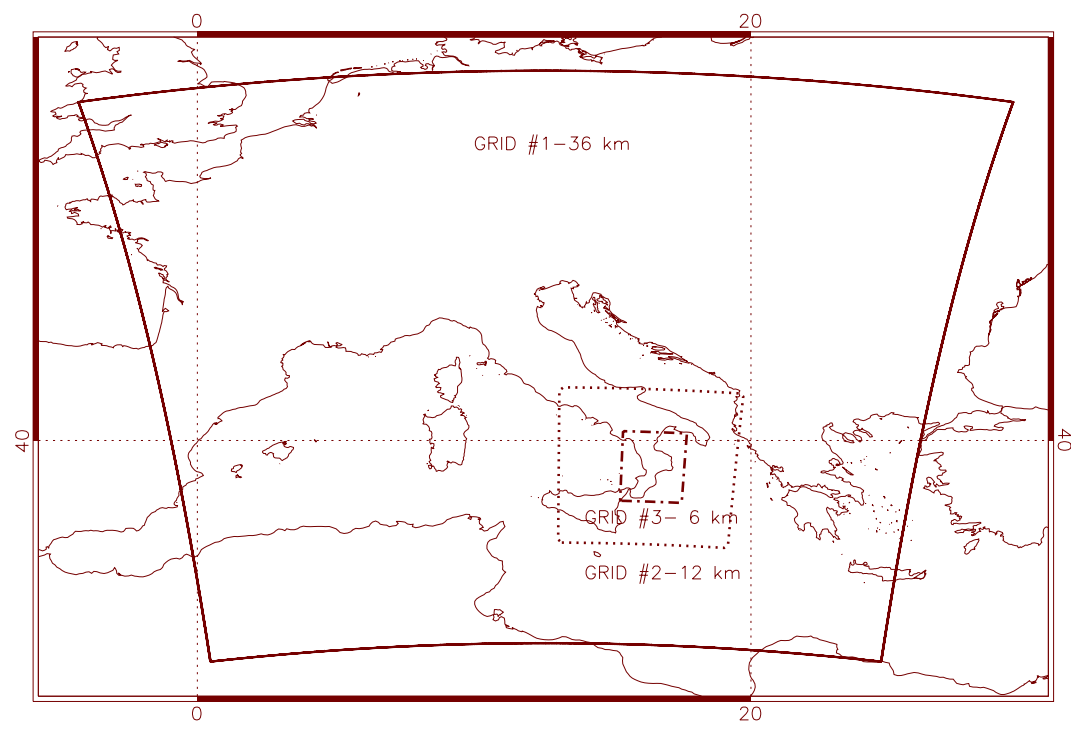

Fig. 2. Simulation domains. Horizontal grid resolutions are $36 \mathrm{~km}, 12 \mathrm{~km}, 6 \mathrm{~km}$ for first, second and third domain, respectively. We use first domain for BF ensemble forecasts, first and second domains for LEPS simulations and all domains for Hi-Res forecasts.

6. Steps 4 and 5 are repeated until only five clusters remain.

7. One RM is selected for each of the five clusters by minimizing the ratio between the distance from its own cluster members and the distance from members belonging to other clusters.

8. Finally, RAMS simulations are performed using RMs as initial and dynamic boundary conditions.

Compared to previous works (Molteni et al., 2001; Marsigli et al., 2001; Montani et al., 2003) this paper does not contain a new methodology. Nevertheless it presents new data for a different geographical area, using different horizontal resolutions and a different Limited Area Model (LAM). The effects of ensemble size reduction and enhanced horizontal resolution are assessed for all case studies selected comparing the Brute Force (BF) and LEPS outputs, an experiment that has not been assessed so much in referenced works. Moreover the topic is of considerable current interest and there is a tendency in the NWP community toward very high horizontal resolution $(<10 \mathrm{~km})$. The value of very high horizontal resolution modelling compared to LEPS has not been assessed and this paper adds new data and results by comparison between High Resolution (Hi-Res) deterministic forecasts and LEPS results.

\section{Model configuration and experimental procedure}

In this paper we use RAMS 4.3 in its non-hydrostatic compressible form (Pielke et al., 1992; Cotton et al., 2003).
Figure 2 shows three domains which are used in this paper. Hereafter we will refer to these as domains \#1, \#2, and \#3 and to their associated grids as the first, second and third grids.

The LEPS configuration has two nested grids with horizontal resolutions of $36 \mathrm{~km}$ and $12 \mathrm{~km}$. They cover the two outermost domains of Fig. 2. Nested grids use a two-way communication method described in Walko et al. (1994), which scheme works as follows. Communication from the parent to the nested grid is accomplished immediately following a time step on the parent grid which updates the prognostic fields. The updated coarse grid values are interpolated sequentially in the coordinate directions to the locations where they are defined on the boundaries of the nested grid. The nested grid boundary values are replaced by these interpolated values. A nested grid usually runs with a shorter time step than the parent grid. Thus, its boundary values are also interpolated linearly in time from the coarse for intermediate nested grid time steps. The nested grid is then updated in a series of smaller time steps until it has caught up to the simulation time of the parent grid. At this time the reverse communication is accomplished by averaging the prognostic variables over each set of nested grid cells which occupy a single parent grid cell and replacing the parent grid cell value with that average. This two-way communication ensures a continuous balance between parent and nested meteorological fields. Apart from specific differences in interpolating functions, the basic difference between one-way and two-way nesting is in the reverse communication which is not performed for the one-way nesting. Several numerical experiments performed with RAMS and other atmospheric models (Walko et al., 1994) show a significant improvement in the solution when two-way nesting is applied. 
Table 1. Selected case studies.

\begin{tabular}{lrl}
\hline EVENT \# & DATE & STORM TYPE \\
\hline 1 & 2.10 .2000 & Atlantic cyclone from Southwest (Saharan Storm). \\
2 & 28.12 .2000 & Atlantic cyclone from Northwest (Rhone valley). \\
3 & 14.1 .2001 & Atlantic cyclone from Southwest (Saharan Storm). \\
4 & 31.3 .2001 & Cyclogenesis on the lee-side of the Alps. \\
5 & 16.12 .2001 & Atlantic cyclone from Northwest (Rhone valley)+Cyclone over Balkans. First cyclone \\
& & produced little precipitation and largest rainfall was produced by the cyclone over Balkans. \\
6 & 24.12 .2001 & Cyclone over Balkans. \\
7 & 4.4 .2002 & Air mass confluence from Balkans and Atlantic. \\
8 & 25.5 .2002 & Cyclogenesis on the lee-side of the Alps. \\
\hline
\end{tabular}

In order to assess the improvements introduced by LEPS higher horizontal resolution, we compare its performance with the BF for all case studies selected in this paper. For $\mathrm{BF}$, RAMS simulations have $36 \mathrm{~km}$ horizontal grid spacing and are nested in all 51 ECMWF-EPS runs. The BF domain is the same as the LEPS first grid. Even if LEPS accounts for cluster population, there is a large reduction of ECMWFEPS members with this technique, so these simulations give an assessment of the relative impact on Quantitative Precipitation Forecasts (QPF) of enhanced horizontal resolution and ensemble size reduction.

Our definition of BF differs slightly from that adopted in previous papers where BF resolution is the same as LEPS. In our case BF uses the first LEPS grid only and the acronym BF refers to the fact that we are dealing with a full 51-member ensemble rather than to its resolution.

In addition to BF and LEPS forecasts, for each case study, we perform a high horizontal resolution deterministic run using all domains shown in Fig. 2. Horizontal resolution for the third grid is $6 \mathrm{~km}$.

We use thirty vertical levels up to $16000 \mathrm{~m}$ in the terrain following coordinate system. Levels are not equally spaced: within the PBL (Planetary Boundary Layer) layers run about 50-200 $\mathrm{m}$ thick, whereas in the middle and upper troposphere they are $1000 \mathrm{~m}$ thick.

Parameterization of the surface water and energy budgets and fluxes with the atmosphere are described in Walko et al. (2000).

Non-convective precipitation is computed from explicit prognostic equations for eight water categories: total water, cloud particles, rain, pristine ice, snow, aggregates, graupel, and hail. Convective precipitation is parameterized following Molinari and Corsetti (1985) who proposed a simplified form of the Kuo scheme that accounts for updrafts and downdrafts. Convection parameterization is applied to the first grid only for BF, LEPS and Hi-Res.
Acceptable numerical resolution of deep convection requires a grid spacing not coarser than about $1 \mathrm{~km}$, which implies that on coarser grids, such as used in the present study, some form of convective pameterization is needed. However, existing convective parameterization schemes are designed for grid spacing larger than about 10 or $20 \mathrm{~km}$ and do not perform properly on meshes finer than this. The RAMS bulk microphysics parameterisation is designed to emphasize individual microphysical processes rather than the statistical end result of atmospheric systems and is intended to be applicable to any atmospheric system (e.g. stratiform or convective clouds, tropical or arctic clouds etc.). However, for it to perform correctly for deep convection, the host dynamic model must adequately resolve the convective motion. As a consequence, a deficiency exists for adequately representing deep convection in this and other numerical models whose grid spacing is between about 1 and 10 to $20 \mathrm{~km}$. For this reason, we did not activate convective parameterization on the second grid, and have instead relied on orographic lifting and vertical motion in resolved larger-scale systems to provide dynamic support for precipitating systems. This choice may limit the applicability of the results presented in this work to situations in which deep convective-scale motion is significant.

To verify forecasts, we use a subjective analysis and objective scores. Subjective evaluation is done by a qualitative comparison, for selected case studies, of probability maps and total precipitation maps with raw data from the raingauge regional network. This is discussed in Sect. 4.1. Objective verification is done computing objective probabilistic and deterministic scores from model output and raingauge measurements for all case studies. The methodology adopted to compute scores is discussed in Sect. 4.2.

Not all raingauges were working for all events, and the number of reporting stations ranges from 35 to 75 depending on the case study. Figure 1 shows raingauge locations. 
Case studies are selected by requesting that at least 10 stations reported more than $30 \mathrm{~mm} /$ day. The Calabria climate is rather dry, and from October 2000 to May 2002, the time frame covered by our database, we found eight case studies. They are reported in Table 1 and are associated with typical storms that produce large and widespread rainfall over Calabria.

In summary three kinds of RAMS simulations are performed for each case study: (a) BF that has one domain with $36 \mathrm{~km}$ horizontal resolution; (b) LEPS that has two two-way nested domains with $12 \mathrm{~km}$ maximum horizontal resolution; (c) Hi-Res that has three two-way nested domains and $6 \mathrm{~km}$ maximum horizontal resolution.

\section{Results}

\subsection{Subjective analysis}

In order to evaluate enhanced performance of LEPS due to its horizontal resolution, we use a subjective analysis for LEPS and BF for two case studies. These are an Atlantic cyclone from the southwest on 14 January 2001 (Event \#3) and cyclogenesis on the lee side of the Alps on 25 May 2002 (Event \#8). The first case study is well represented by BF and by LEPS, while the second event is less satisfactory. These case studies are representative of the performance spread that is obtained for the events selected.

Figure 3 shows raingauge measurements for the 14 January 2001 case study. Several stations reported more than $100 \mathrm{~mm}$, and the maximum rainfall recorded is about $150 \mathrm{~mm}$. There are two main effects produced by local orography on precipitation. First, rainfall is higher over elevated peaks as a consequence of the orographic uplift. Second, it is larger over the eastern half of Calabria due to the orographic shield. Indeed, for this storm, air masses crossed Calabria from South-East leaving more abundant rainfall on the upwind slope of mountains. It is worth noting that almost all raingauges on the East side of Calabria reported more than $50 \mathrm{~mm}$.

Figure 4a shows total precipitation simulated by BF considering the whole event duration. This map reports the ensemble mean (EM). Because EM averages out small scale unpredictable detail, it does not show extreme precipitation totals. There is a large area where rainfall is greater than $10 \mathrm{~mm}$. This area reflects the storm path that entered from the Strait of Gibraltar and then moved from west to east leaving a precipitation swath. During this kind of synoptic pattern evolution, a low pressure centre is well developed over the southwestern Mediterranean basin and this configuration forces advection of warm and moist air masses from the southeast toward Calabrian Ionian coasts. This basic situation and the humid-warm advection are a rather common pattern in cases of intense and abundant precipitation over the Ionian Calabrian coast (Federico et al., 2003). Forecast rainfall is

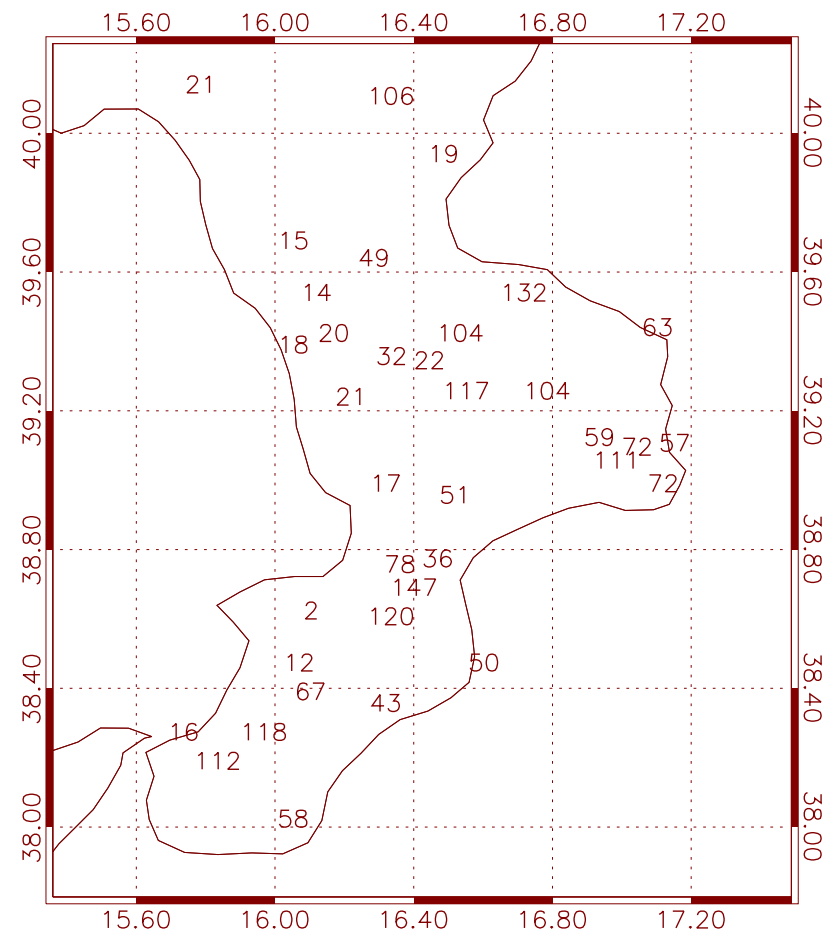

Fig. 3. Raingauge measurements (mm) for 14 January 2001 (cumulated rainfall from 12 January - 23:00 UTC to 14 January 22:00 UTC).

greater than $30 \mathrm{~mm}$ over eastern Calabria with some areas exceeding $50 \mathrm{~mm}$.

Figure $4 \mathrm{~b}$ shows precipitation for the same area as Fig. 4a but for the LEPS first grid and it shows EM too. For consistency we use the first LEPS grid output to derive precipitation and probability fields and to plot these fields on the same BF subdomain. The effects of the inner LEPS grid are transferred to the parent grid by the two-way interactive nesting. Figure $4 \mathrm{~b}$ shows the LEPS weighted mean precipitation and the weights are assigned according to RM cluster population. Due to the higher horizontal resolution and the two-way nesting, orographic forcing is better represented in LEPS, compared to BF, and a larger precipitation amount is simulated over eastern Calabria. In particular, for the second LEPS grid (not shown), rainfall is larger than $90 \mathrm{~mm}$ over the main peaks and in large areas of eastern Calabria.

One of the key issues with EPS is to cast the forecast in a probabilistic form. Figures $5 \mathrm{a}$ and $\mathrm{b}$ show, respectively, the probability of precipitation larger than $50 \mathrm{~mm}$ for the BF and LEPS first grid, respectively. The LEPS probability is obtained weighting RM probabilities ( 0 or 1 ) according to cluster population. From Fig. 5a there are hints of large rainfall; probability larger than $50 \%$ is forecast for eastern Calabria and there is a clear suggestion of large precipitation amounts associated with this storm. Due to the higher horizontal resolution and to the two-way nesting used in RAMS, the LEPS 


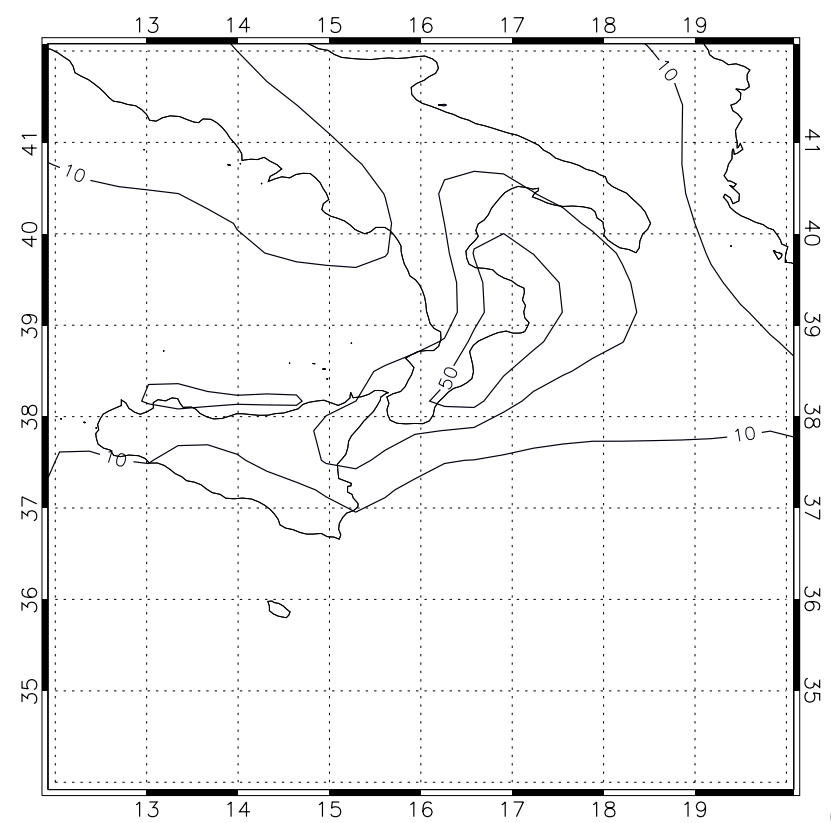

(a)

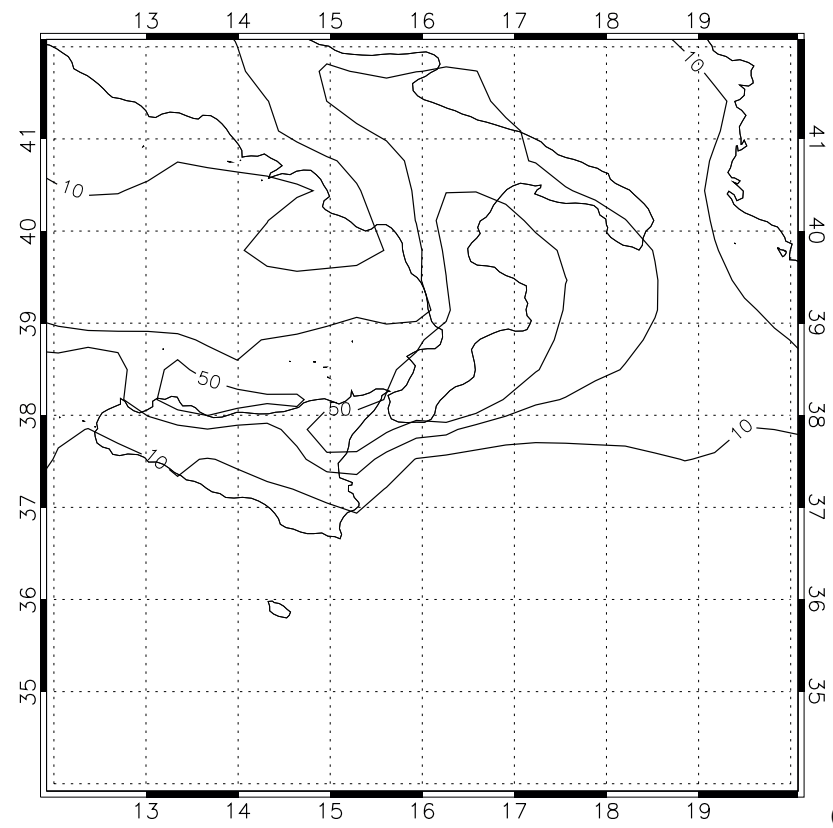

Fig. 4. (a) BF EM precipitation for 14 January 2001 (cumulated from 12 January - 23:00 UTC to 14 January - 22:00 UTC). Contours are for $10 \mathrm{~mm}, 30 \mathrm{~mm}$ and $50 \mathrm{~mm}$. Simulations start on $11 \mathrm{Jan}-$ uary 2001, 12:00 UTC. The map is plotted for a subdomain centred in Southern Italy for clarity. (b) As in Fig. 4a but for LEPS first grid ensemble weighted mean precipitation. Weights are assigned according to RM cluster population. The map is for LEPS first domain and it is plotted for a subdomain centred in Southern Italy for clarity. first grid, Fig. 5b, shows a probability larger than $50 \%$ over all eastern Calabria and LEPS gives an even clearer suggestion of the storm severity compared to BF.

To better appreciate the effects of enhanced horizontal resolution on QPF for this severe event, we plot the probability of more than $100 \mathrm{~mm}$ rainfall for the LEPS second grid in Fig. 5c. Values greater than $50 \%$ and $80 \%$ are shown over Serre and Sila, respectively (see Fig. 1). Results compare well with measurements (Fig. 3) and give a good forecast of the storm severity. The BF forecast (not shown) over the same area gives values less than $10 \%$ everywhere. This result highlights the role of local physiographic features and horizontal grid resolution for QPF over Calabria.

Even if, for this case study, there are evident signals of intense and abundant rainfall from BF, LEPS highlights better precipitation field features over Calabria, both in terms of probability and total precipitation. Comparing Figs. 4a/5a and Figs. $4 \mathrm{~b} / 5 \mathrm{~b}$ it is noticeable that LEPS gives significant precipitation around the North coast of Sicily. Unfortunately, data are not available to us to verify this point.

Figure 6 shows raingauge measurements for 25 May 2002. Larger amounts were recorded in eastern and southern Calabria and over two of the five mountainous areas (Serre and Aspromonte). This reflects again the shield effect due to orographic barriers and rainfall uplift enhancement. Indeed, during the storm evolution, air masses crossed Calabria mainly from the southeast so larger precipitation was recorded on the upwind side of the mountains and over the main peaks.

Despite the storm intensity, BF and LEPS performances are unsatisfactory for this event. In particular the BF-EM precipitation map (not shown) has values less than $10 \mathrm{~mm}$ over Calabria. Probabilities of rainfall larger than $10 \mathrm{~mm}$ and $20 \mathrm{~mm}$ are, respectively, less than $20 \%$ and $10 \%$ over the peninsula, so the $\mathrm{BF}$ approach gives no signal for this severe case study.

LEPS results are also unsatisfactory, but there are improvements due to the enhanced horizontal resolution. Figure $7 \mathrm{a}$ shows the LEPS first grid cumulated precipitation. Contours are for $10 \mathrm{~mm}$ and $30 \mathrm{~mm}$. Values larger than $30 \mathrm{~mm}$ are forecast over southern Calabria and values larger than $10 \mathrm{~mm}$ are predicted almost everywhere over the peninsula. Hints for a moderate storm come also from Fig. $7 b$ that reports the probability of precipitation larger than $30 \mathrm{~mm}$. Values greater than $30 \%$ are forecast for southern Calabria.

It is worth noting that the LEPS second grid has clearer and more defined signals for a moderate-intense event. In particular, the probability of more than $30 \mathrm{~mm}$ (not shown) is larger than $50 \%$ over southern Calabria and Sila; moreover, for the LEPS second grid, the probability map for $50 \mathrm{~mm}$ threshold (not shown) indicates values greater than $10 \%$ over southern Calabria. Apart from larger values simulated by the LEPS second grid, it is also important to note that structures and mesoscale patterns are more refined for the LEPS second grid, information that would be useful to the forecaster. 

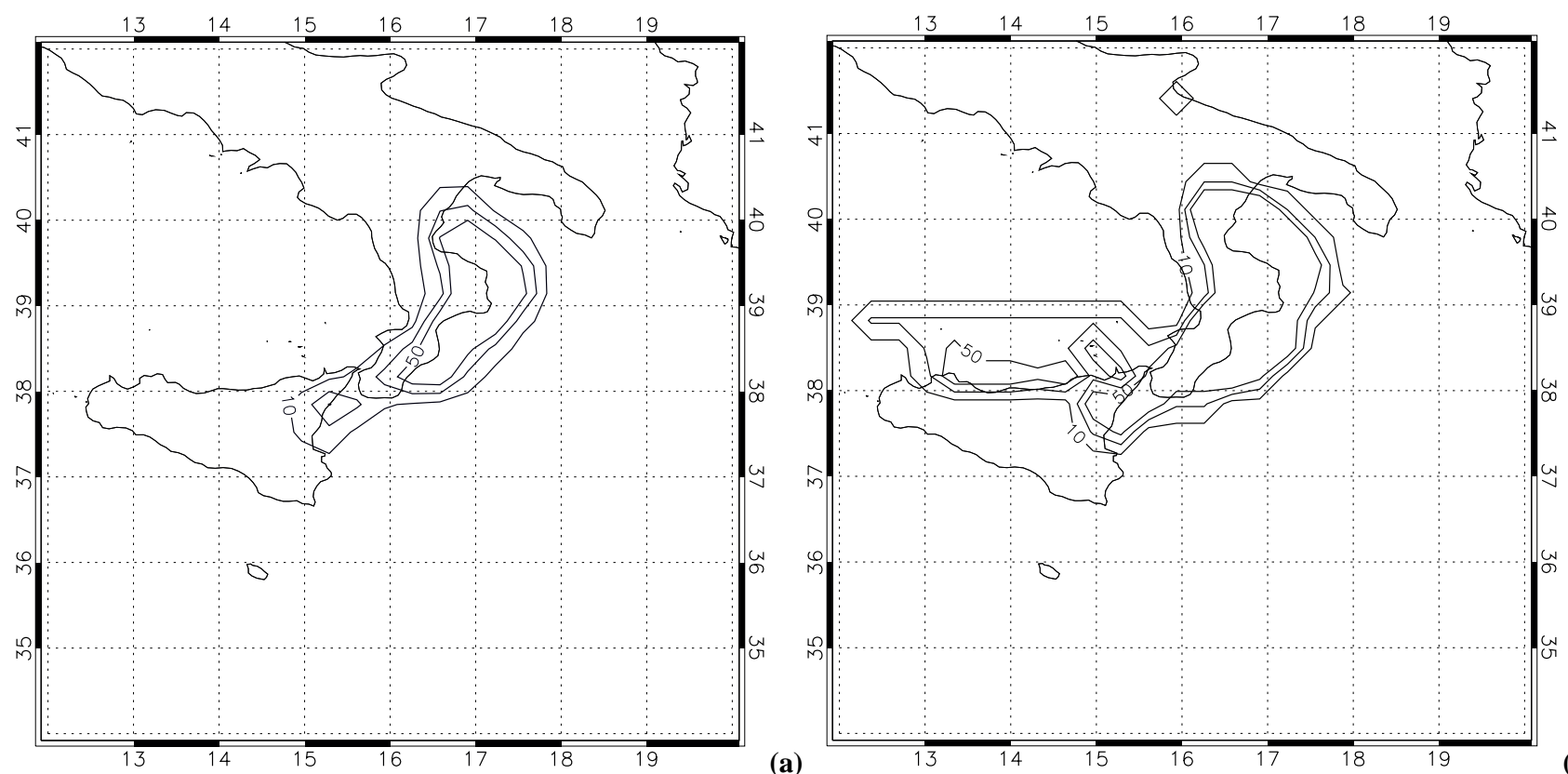

(b)

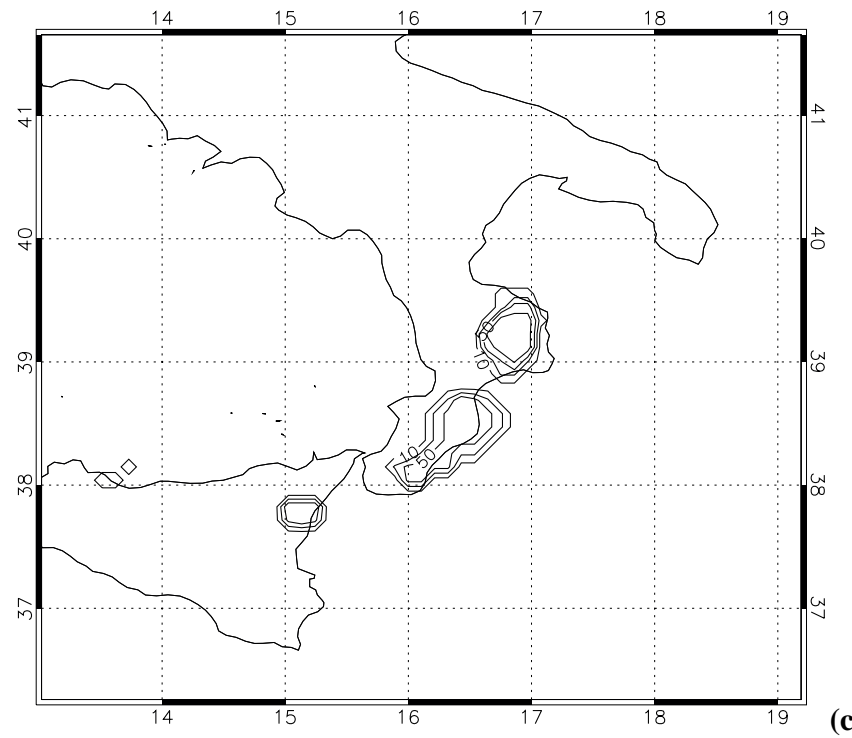

Fig. 5. (a) BF probability of more than $50 \mathrm{~mm}$ rainfall for 14 January 2001 . Contours are reported for $10 \%$, 30\% and $50 \%$. Precipitation has been cumulated from 12 January - 23:00 UTC to 14 January - 22:00 UTC. Simulations start on 11 January 2001 - 12:00 UTC. The map is plotted for a subdomain centred in Southern Italy for clarity. (b) As in Fig. 5a but for LEPS first grid. RM precipitation has been cumulated from 12 January - 23:00 UTC to 14 January - 22:00 UTC. Weights are assigned to RM probability fields (0 or 1) according to cluster population. Simulations start on 11 January - 12:00 UTC. The map is for LEPS first domain and is plotted for a subdomain centred in Southern Italy for clarity. (c) As in Fig. 5b but for LEPS second grid and for $100 \mathrm{~mm}$ threshold. Contours: $10 \%, 30 \%, 50 \%, 80 \%$.

To further explore the role of enhanced horizontal resolution for this case study and to make better use of LEPS simulations when severe events are suspected to occur, we show the maximum for the LEPS second grid in Fig. 7c. This map can be useful to the skilled forecaster but must be used carefully because in operations it can lead to overestimation.
Precipitation larger than $50 \mathrm{~mm}$ is forecast over southern Calabria where the map is much more representative of storm severity compared to Fig. 7a. At the same time this result shows that LEPS-EM cannot be expected to represent extreme precipitation totals because EM averages out small scale unpredictable components. This point must be 


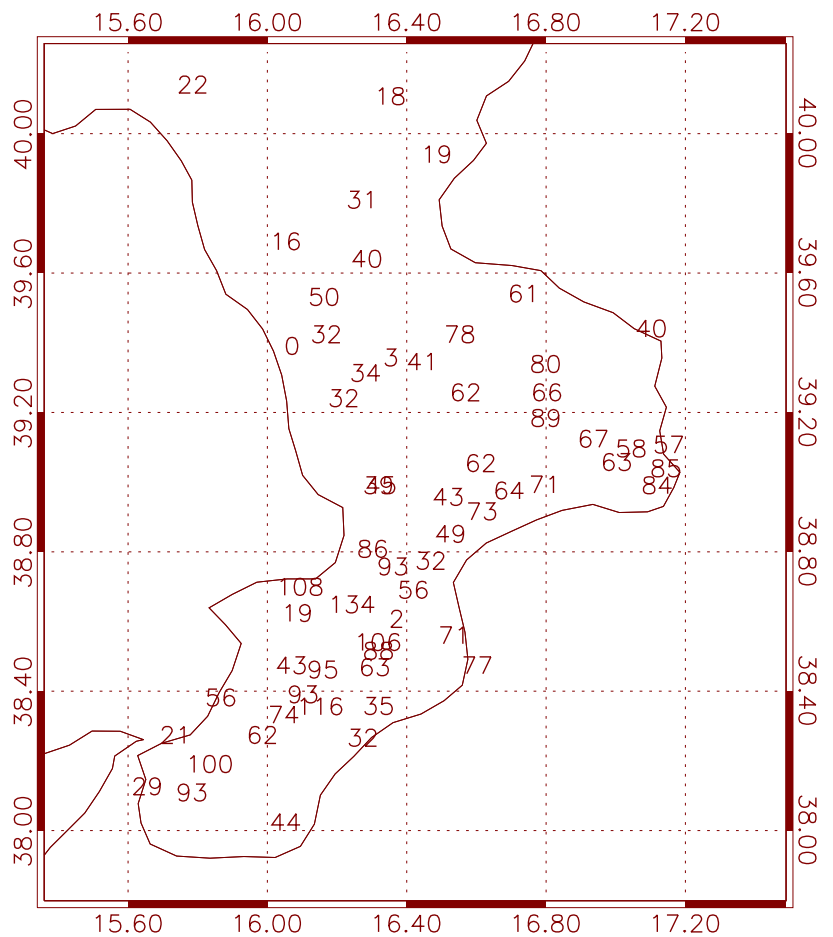

Fig. 6. Raingauge measurements (mm) for 25 May 2002 (cumulated from 23 May - 23:00 UTC to 25 May - 22:00 UTC).

considered when comparing LEPS-EM and Hi-Res objective scores in the next subsection. Despite the better representation of storm severity there is still an evident rainfall underestimation.

Comparison of LEPS results and raingauge measurements shows that simulated precipitation is underestimated, mainly for higher thresholds. However, compared to BF, LEPS gives suggestion of a moderate-intense event.

Precipitation amounts and the convective environment are better forecast by Hi-Res. Figure 7d shows total rainfall for this simulation. Precipitation is larger than $70 \mathrm{~mm}$ over south-western Calabria, mainly due to orographic uplift of Serre and Aspromonte, and more than $100 \mathrm{~mm}$ are forecast on the North-West side of Aspromonte. Performance is less satisfactory for the northern half of Calabria where rainfall is lower than $50 \mathrm{~mm}$. For this area performance is comparable with LEPS maximum precipitation. Note that with its enhanced horizontal resolution, Hi-Res captures better than LEPS the mesoscale and finer scale structures, such as the shielding effect of Serre and Aspromonte and the precipitation pattern over Sila and Catena Costiera, giving results that are closer to observations.

The behaviour discussed for the above two case studies is obtained for all events; i.e. there is a clear improvement of rainfall forecast fields when LEPS is compared to BF, both in terms of probability maps and total precipitation. Considering also that CPU time required to run LEPS is less than for
Table 2. Contingency table of possible events.

\begin{tabular}{|c|c|c|c|}
\hline & \multicolumn{3}{|c|}{ OBSERVED } \\
\hline & & YES & NO \\
\hline & YES & $\mathrm{a}$ & b \\
\hline FORECAST & NO & $\mathrm{c}$ & d \\
\hline
\end{tabular}

$\mathrm{BF}$, we conclude that, for our case studies, it is better to run a high resolution ensemble with fewer members than the whole BF. For cases with larger spread and for which local forcing is less important, BF performance should improve compared to LEPS and the impact of reduction of members should be larger. Our cases are limited to intense and abundant precipitation where mesoscale and local scale play a fundamental role and horizontal grid resolution is a key factor for improving the forecast.

\subsection{Objective deterministic and probabilistic scores}

Although Calabria has a relatively dense raingauge network, the number of reporting stations and the terrain complexity do not allow for a reliable objective analysis of raingauge data. Therefore, we use the following methodology for the objective verification. First, remapping is performed for all RAMS configurations on a common $0.1^{\circ} \times 0.1^{\circ}$ grid following the procedure proposed by Baldwin (2000) and used at NCEP (National Centers for Environmental Prediction). The advantage of this method is that it can conserve precipitation to any degree of accuracy. Once remapped, the precipitation forecast is compared with raingauge measurements. Only grid boxes containing one or more raingauges are considered for the comparison. If more than one raingauge is located in a grid box, their average is considered for the comparison. All scores presented in this paper refer to the whole event, i.e. considering the total rainfall cumulated since the end of spin up time which is $11 \mathrm{~h}$.

Objective scores for LEPS can be calculated starting from the precipitation field on the first or second grid; similarly, scores for Hi-Res can be calculated starting from the first, second or third grid outputs. In order to better represent the effect of enhanced horizontal resolution, we compute objective scores reported in this subsection from the highest resolution outputs, i.e., the second grid for LEPS and the third grid for Hi-Res. However, the two-way interactive nesting transfers local and mesoscale features from the inner to the parent grid and results are qualitatively similar for scores derived from different grids of the same model configuration.

An ensemble system can provide both deterministic and probabilistic forecasts. Indeed each ensemble member represents a deterministic forecast but the whole ensemble can be used to compute probabilities and can be validated in terms 

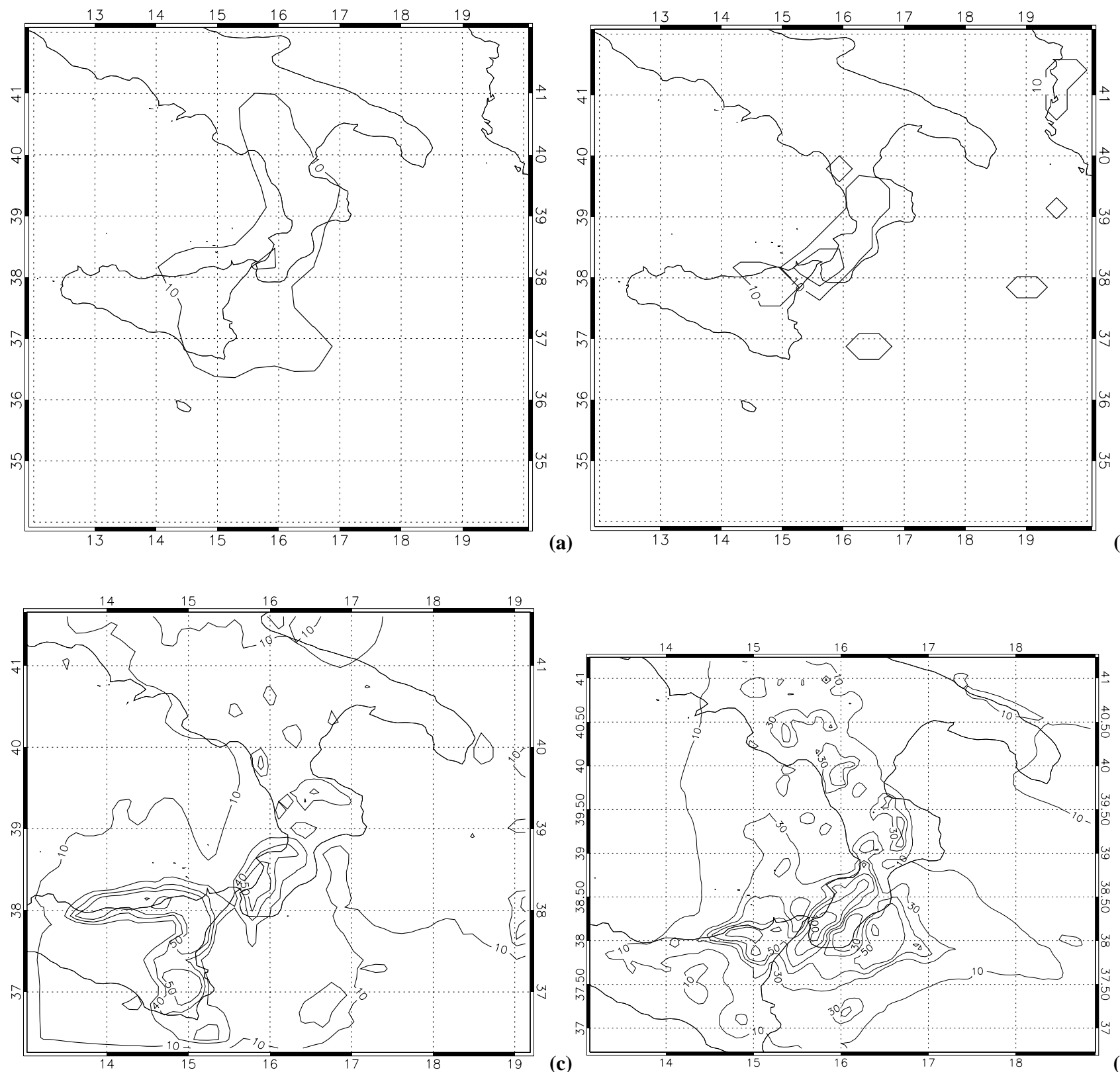

(d)

Fig. 7. (a) LEPS first grid ensemble weighted mean precipitation for 25 May 2002 (cumulated from 23 May - 23:00 UTC to 25 May 22:00 UTC). Contours are for $10 \mathrm{~mm}$ and $30 \mathrm{~mm}$. Simulations start on 22 May 2002, 12:00 UTC. The map refers to LEPS first domain and is plotted for a subdomain centred in Southern Italy for clarity. (b) LEPS first grid probability of more than 30 mm rainfall for 25 May 2002. Contours are reported for $10 \%$ and 30\%. Precipitation has been cumulated from 23 May - 23:00 UTC to 25 May - 22:00 UTC. Weights are assigned to RM probability fields according to cluster population. Simulations start on 22 May 2002 - 12:00 UTC. The map refers to the LEPS first domain and is plotted for a subdomain centred in Southern Italy for clarity. (c) Maximum total rainfall for LEPS second grid for 25 May 2002. Precipitation has been cumulated from 23 May - 23:00 UTC to 25 May - 22:00 UTC and for each grid point the maximum among RMs has been selected. Contours are for 10, 30, 40 and $50 \mathrm{~mm}$. (d) Hi-Res third grid precipitation forecast for $25 \mathrm{May}$ 2002 (cumulated from 23 May - 23:00 UTC to 25 May - 22:00 UTC). Contours are reported for 10, 30, 40, 50, 70 and 100 mm.

of probabilistic scores. A thorough review of scores used in this paper can be found in Wilks (1995), Mason and Graham (1999) and Stanski et al. (1989).
Deterministic scores analyzed are the bias (BIA) and equitable threat score (ETS). To compute these scores, precipitation space is divided in four mutually exclusive and exhaustive sets (contingency table, Table 2): hits (a) represent 


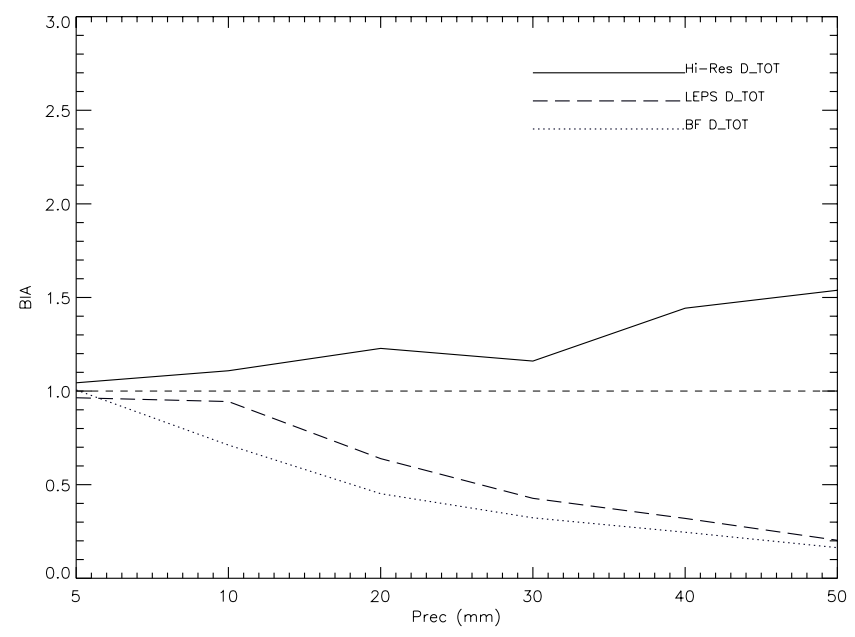

Fig. 8. BIA score for Hi-Res (solid line), LEPS (dashed line) and $\mathrm{BF}$ (dotted line) as a function of rainfall threshold and averaged over all case studies. LEPS results are derived starting from second grid output, Hi-Res results are derived starting from third grid output. LEPS and BF are ensemble weighted means.

occasions where both rain forecasts and measurements are greater than or equal to a threshold; false alarms (b) represent the number of locations where the model is above a threshold whereas measurement is under the same threshold; misses (c) represent occasions where the measurement is above a threshold and forecast is under the same threshold; correct no forecasts (d) represent occasions where the model and measurements are both under the threshold.

Starting from the contingency table we define BIA:

$B I A=\frac{a+b}{a+c}$

BIA measures if the model over-forecasts or under-forecasts precipitation frequency over an area for a selected threshold. If BIA $>1$ the model overestimates the precipitation area, and if $\mathrm{BIA}<1$ the model underestimates this area. For a perfect forecast $\mathrm{BIA}=1$.

The most widely used score is Equitable Threat Score (ETS) defined as:

$$
E T S=\frac{a-a_{r}}{a+b+c-a_{r}}
$$

where $a_{r}$ is the expected number of correct forecasts above the threshold in a random forecast where forecast occurrence/non-occurrence is independent from observation/non-observation. It is defined as:

$a_{r}=\frac{(a+b)(a+c)}{a+b+c+d}$

For a perfect forecast ETS is equal to 1, while it is less than or equal to zero for a useless forecast.

Probabilistic scores used in this paper are relative operating characteristic (ROC) area (Mason and Graham 1999) and
Brier score (Stanski et al., 1989; Wilks 1995). From contingency Table 2 we define HR (Hit Rate) and FAR (False Alarm Rate) as follows:

$$
\begin{aligned}
& H R=\frac{a}{(a+c)} \\
& F A R=\frac{b}{(b+d)}
\end{aligned}
$$

They represent, respectively, the proportion of events that were actually forecast and the proportion of non-events that were incorrectly forecast to occur. A useful measure for assessing the ability of probabilistic scores to discriminate dichotomous events, and which has been proposed to compare deterministic and probabilistic systems, is the ROC that utilizes information from the contingency Table 2. For a probabilistic forecast, a set of contingency tables can be constructed for a fixed threshold using different probabilities as decision criteria, ranging from $0 \%$ (i.e. an event is always forecast) to $100 \%$ (i.e. an event is never forecast). Plotting HR against FAR over the range of decision criteria forms the ROC. A perfect forecast system yields $H R=1$ and $F A R=0$, represented by the upper left corner of the ROC diagram. A completely unskillful forecast system is unable to distinguish between event occurrence/non-occurrence (HR=FAR) and ROC lies along the diagonal from the point $(0,0)$ to $(1,1)$.

Forecast quality is often summarized in terms of the area (A) under the ROC curve, with a skilful system having A significantly greater than 0.5 .

The Brier score is defined as the average of squared differences between forecast probabilities and the corresponding binary values representing occurrence/non-occurrence of the event. It is a negatively oriented score: for a perfect forecast $\mathrm{BS}$ is equal to zero.

It is not straightforward to summarize the performance of LEPS for the eight cases study considered in this paper by objective scores. The whole experimental setup is not large enough for a reliable statistical evaluation, and cases span situations with different predictability. A statistical evaluation of the model setups utilized in this work will be the subject of future studies. Nevertheless, objective scores can be used to compare the relative performance of different model configurations reported in this paper. They are presented as averages over all case studies. Even if there is a spread among different cases, objective score averages give a summary of the performance of model setups and are useful to quantify their differences. However, results of this paper are drawn from individual cases and may not be applicable in different circumstances.

The reader should also be aware that scores presented in this paper refer to selected events characterized by moderateintense precipitation that are not representative of the model general behaviour. Federico et al. (2004) reports performance for RAMS Hi-Res deterministic forecasts for a two year period. Nevertheless, objective scores reported for selected cases, such as moderate-intense precipitation, are useful to evaluate model setups under those conditions and help 


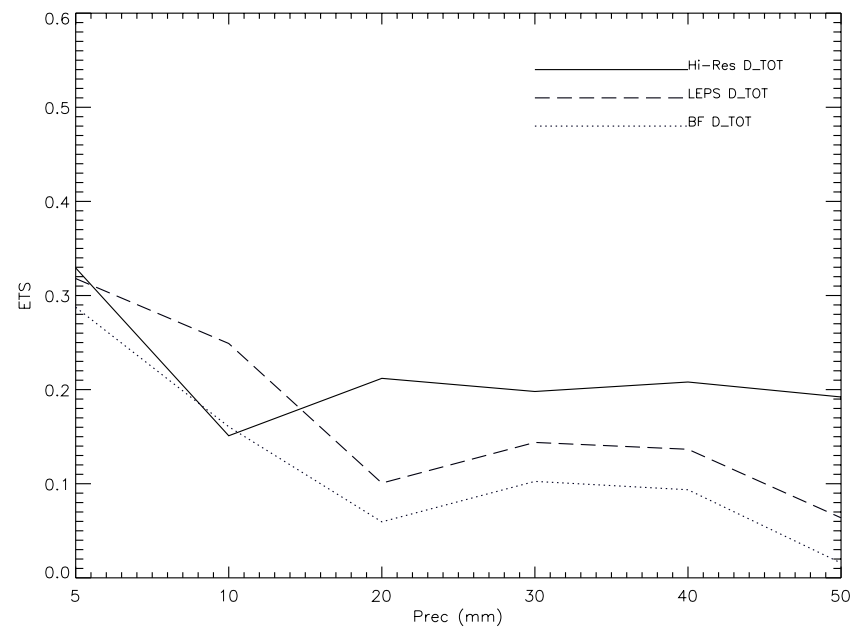

Fig. 9. As in Fig. 8 for ETS. LEPS and BF are ensemble weighted means.

to isolate key issues to be changed for a better forecast. The skilled forecaster has useful hints about the behaviour of a model setup from objective scores when a particular event (such as moderate-intense precipitation) is likely to occur. Thus, objective scores can be a valid tool to exploit in order to issue a better forecast even when they are computed for selected cases.

Figure 8 reports the BIA score for different rainfall thresholds for Hi-Res, LEPS and BF. With respect to this comparison for BIA and ETS (Figs. 8 and 9), notice that LEPS and BF curves do not show extreme precipitation totals because EM averages out small scale unpredictable components, and it is expected that the Hi-Res forecast will perform better at least for the largest thresholds. Figure 8 does indicate that Hi-Res has the best performance for thresholds larger than $20 \mathrm{~mm}$, but LEPS-EM performs better for a $10 \mathrm{~mm}$ threshold. LEPS-EM underestimates precipitation amounts and this behaviour is evident for precipitation larger than $10 \mathrm{~mm}$. HiRes has the opposite behaviour, i.e. it overestimates precipitation areas, mainly for larger thresholds. BF-EM has the worst performance and BIA values reflect the absence of local scale and mesoscale forcing that enhance rainfall through several mechanisms (Smith 1979; Federico et al., 2003).

Figure 9 shows results for the ETS score. We note that $\mathrm{Hi}$-Res and LEPS-EM are useful (ETS $>0$ ) for all thresholds considered in this paper, and BF-EM has the worst performance. This confirms BIA results and shows that BF horizontal resolution is too coarse to represent regional physiographic features properly. Comparing Hi-Res and LEPS-EM, the former performs better for thresholds larger than $10 \mathrm{~mm}$ while LEPS-EM has a better score for the $10 \mathrm{~mm}$ threshold.

Figure 10 shows results for the ROC area. Main conclusions are similar to those discussed for ETS and BIA. Indeed BF horizontal resolution is too coarse to represent local physiographic forcing, and performance decreases with

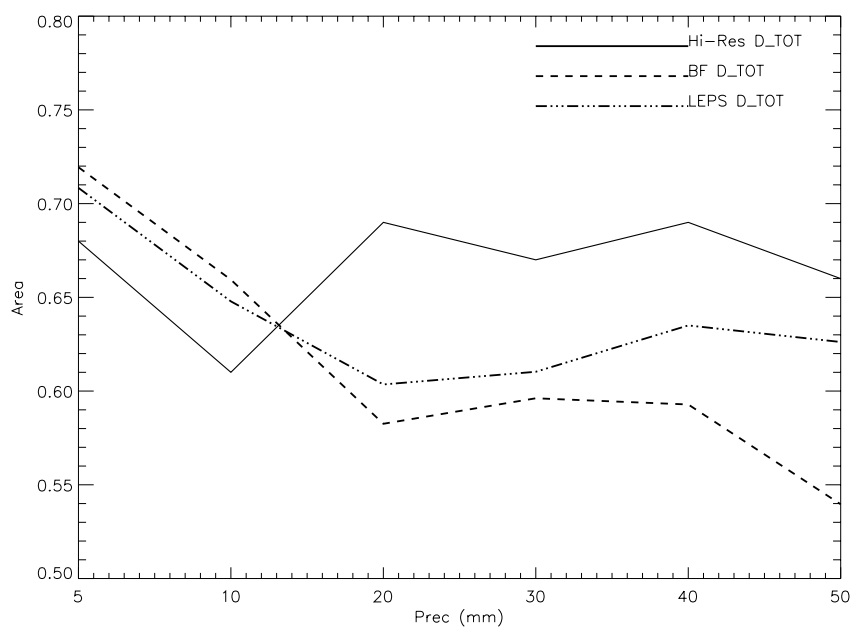

Fig. 10. As in Fig. 8 for the ROC area. Results for LEPS are derived from the second grid output. Hi-Res results are derived from the third grid output.

increasing rainfall. LEPS and BF have a comparable ROC area up to a $10 \mathrm{~mm}$ threshold. For larger thresholds LEPS outperforms BF. LEPS performs better than Hi-Res up to a $10 \mathrm{~mm}$ threshold, then its performance decreases and the score is better for Hi-Res.

Figure 11 shows the Brier Score for BF and LEPS as a function of rainfall threshold. LEPS always performs better than $\mathrm{BF}$, and differences are larger for intermediate rainfall thresholds.

Previous results for objective scores confirm the need of higher resolution compared to currently available EPS for Calabria, in order to better represent mesoscale and local scale forcing.

We conclude this discussion on LEPS by considering the relationship between ensemble spread, quantified by the $700 \mathrm{hPa}$ geopotential height, and LEPS performance. Indeed, one of the appealing EPS feature is its ability to relate forecast skill to the ensemble spread. Figure 12 shows the $700 \mathrm{hPa}$ geopotential spread, i.e. the standard deviation from EM, averaged over all EPS members, versus forecast time. Spread is computed from ECMWF-EPS members and little CPU time is required to quantify this parameter. Figure 12 suggests that case studies can be combined in three groups. The first one has the lowest spread and contains two cases: 28 December 2000 and 14 January 2001. The second group has the highest spread and contains the 16 December 2001 case study. The third group contains the remaining cases and has an intermediate spread.

The 14 January 2001 case was discussed in Sect. 4.1 and was classified as one of the best forecasts. It belongs to the first group. The 25 May 2002 case was also discussed in Sect. 4.1 and was considered less satisfactory. It belongs to the third group. A detailed analysis of contingency tables and objective scores reveals that the best results are for 


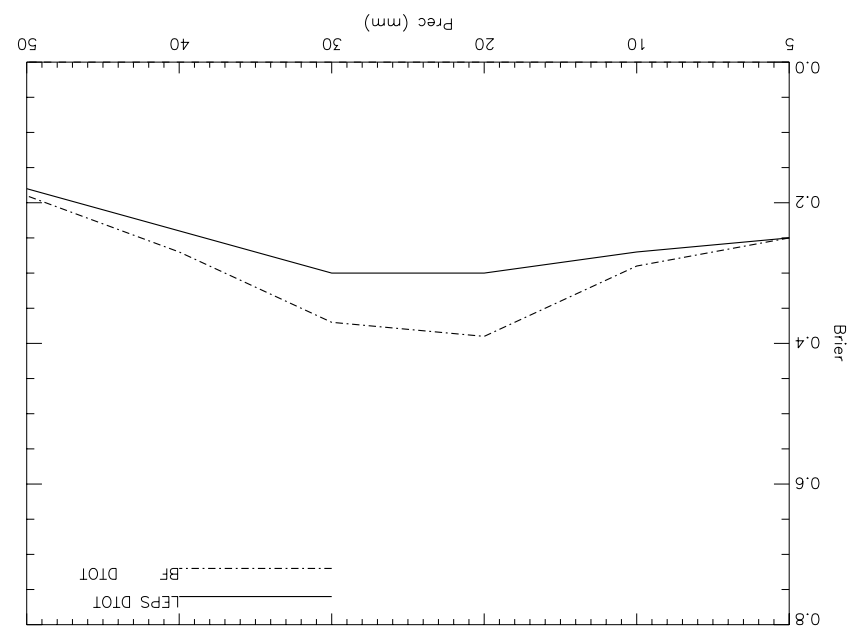

Fig. 11. Brier score for BF (dot-dashed line) and LEPS (solid line) as a function of rainfall threshold and averaged over all case studies. Results for LEPS are derived from the second grid output.

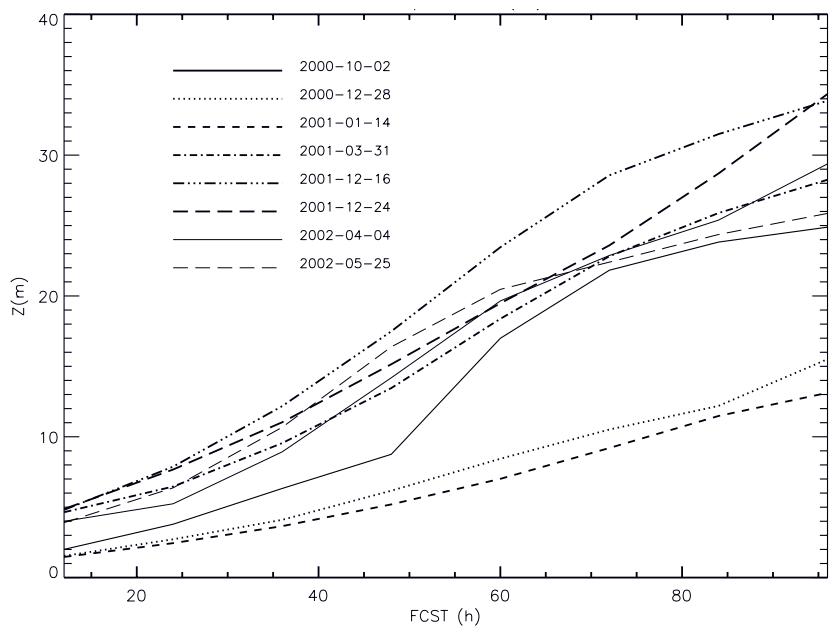

Fig. 12. $700 \mathrm{hPa}$ geopotential height spread for case studies as a function of forecast time. Spread is derived from ECMWF-EPS members and is averaged over the LEPS target area: $5^{\circ} \mathrm{E}-20^{\circ} \mathrm{E}$; $35^{\circ} \mathrm{N}-45^{\circ} \mathrm{N}$.

28 December 2000 and 14 January 2001 cases, i.e. for the first group, while the worst forecast is for the 16 December 2001 case, i.e. the second group. For other cases, scores are similar. It follows that, for storms selected in this study, there is a clear relationship between ECMWF-EPS $700 \mathrm{hPa}$ geopotential spreads and objective scores. This relationship will be investigated further in future studies.

\subsection{Hi-Res and LEPS-EM intercomparison}

Although there is a trend toward higher resolution numerical weather prediction, the value of very high-resolution modelling (here defined as $<10 \mathrm{~km}$ grid spacing) compared to other forecasting methodology as LEPS, has not been assessed. High resolution forecasting appears to be most useful for strongly forced convection (associated with fronts, topography, etc.) but without such forcing it can result in worse scores than a comparatively lower resolution forecast as reported in several studies (Brooks et al., 1992; Colle et al. 2000; Mass et al. 2002). Indeed, the use of high horizontal resolution forecasting usually improves structural definition of mesoscale and local scale features. Nevertheless objective scores can be worse due to spatial and timing errors that are always present. In addition model performance can be reduced, as horizontal resolution increases, due to rainfall overprediction.

Considering objective scores reported in the previous subsection, we can divide performance in two parts: for thresholds $\leq 10 \mathrm{~mm}$, LEPS-EM performs better than Hi-Res, while for larger thresholds, Hi-Res is better.

For rainfall larger than $10 \mathrm{~mm}$ LEPS-EM gives too-low precipitation, and an inspection of the contingency table (not shown) shows that: a) hits are less for LEPS-EM than for HiRes; b) false alarms are less for LEPS-EM than Hi-Res; and c) misses are greater for LEPS-EM than for Hi-Res. Also more (less) hits results in higher (lower) objective scores whereas more (less) misses or false alarms results in lower (higher) objective scores. Indeed, scores are a "summary" of variations in contingency tables stated above. Compared to Hi-Res, for thresholds larger than $10 \mathrm{~mm}$, hits decrease and misses increase, which has a negative impact on LEPSEM objective scores that exceeds the benefits gained by the decrease in false alarms. This indicates that the precipitation forecast by LEPS-EM is too low for these thresholds and suggests that $12 \mathrm{~km}$ grid spacing, currently used by LEPS-EM, is too coarse to represent some important Calabria topographical features.

For thresholds $\leq 10 \mathrm{~mm}$, BIA and ETS results are better for LEPS-EM than for Hi-Res. Inspection of the contingency table (not shown) shows that this is related to Hi-Res rainfall overprediction. The Hi-Res forecast has more false alarms, fewer misses and more hits than LEPS-EM, however the increase in false alarms has a larger impact on scores even though the convective environment is better represented by the Hi-Res forecast.

In summary, with respect to LEPS-EM and Hi-Res forecasts, we conclude that Hi-Res has better objective scores and gives a better representation of the convective environment than LEPS-EM. Nevertheless, unavoidable timing and position errors can have a detrimental effect on scores for lower thresholds.

\section{Conclusions}

We investigated the possibility of forecasting severe weather events by the LEPS methodology over the Calabria Peninsula in southern Italy. 
The aim of LEPS is to transfer advantages of probabilistic forecasting from global to regional scale where localized meteorological systems can produce severe weather and damage. In order to accomplish this task, LEPS uses a limited area model with a much higher horizontal resolution compared to currently available global circulation model EPS.

To save computer time required to run the ensemble, LEPS uses a cluster methodology that, in our case, reduces the whole ECMWF-EPS to five RMs using a hierarchical cluster analysis. Post-processed maps and scores are computed by weighting RM fields according to their own cluster population.

The LEPS methodology, based on RAMS 4.3, was tested for eight case studies. Although we cannot draw any general conclusion due to the limited extent of our database, there are valuable suggestions for future work and for a LEPS operational set-up.

Because of the higher spatial horizontal resolution, LEPS rainfall patterns are closer to the observations compared to the BF approach. This is due to a better representation of local scale and mesoscale forcing. Better LEPS performance is evident by subjective analysis and objective scores. Subjective analysis shows that when large scale forcing is properly represented by ECMWF-EPS, both BF and LEPS performance are satisfactory. Nevertheless LEPS gives more detailed patterns, and precipitation amounts are closer to observations. When large-scale forcing is not well forecast by ECMWF-EPS, BF and LEPS perform poorly. However, LEPS outperforms BF in this case because of the higher horizontal resolution, and it gives indications of moderate-severe weather, at least for case studies selected.

Objective scores, both deterministic and probabilistic, reveal that LEPS outperforms BF for almost all thresholds and for all events. In particular, BF is inadequate to represent local scale and mesoscale forcing, and simulated rainfall is too low. This result poses questions on the utilization of global scale model EPS in regions such as Calabria where local scale and mesoscale forcing is associated with strongly forced convection, and the results of this work suggest that the use of limited area model EPS could be used to tackle this issue.

The relation between ensemble spread, quantified by the $700 \mathrm{hPa}$ geopotential height and computed from ECMWFEPS members to require little additional computational time, is strictly related to LEPS performance. Objective and subjective analyses reveal that case studies with lower spread have better QPFs, whereas events with comparatively larger spread are less satisfactory.

Hi-Res and LEPS-EM comparison shows that Hi-Res tends to overestimate rainfall, while LEPS-EM underestimates precipitation. LEPS-EM scores better for rainfall $\leq 10 \mathrm{~mm}$ but is worse for larger thresholds. For the cases of moderate-intense precipitation considered in this study, we conclude that Hi-Res has better performance. Thus, the use of a high resolution forecast, with grid spacing less than
$10 \mathrm{~km}$, is strongly recommended for Calabria and LEPS. Even higher resolution than used in the present study would be desirable since some mesoscale features were not properly represented.

On the other hand, LEPS gives additional information compared to Hi-Res that is very useful to forecasters. First, it casts the forecast in a probabilistic form, and second, it is able to relate forecast performance to the ensemble spread. We conclude that the optimal approach to forecasting for Calabria would be a hybrid in which LEPS forecasts provide probabilistic forecasts about large-scale evolution and forecast reliability, and a limited number of $\mathrm{Hi}$-Res forecasts give a better representation for the largest precipitation amounts.

Despite the above conclusions, this study is preliminary and work is in progress to acquire data for other case studies in order to compute more reliable statistics.

Other aspects of the LEPS methodology need to be assessed. LEPS reduces ECMWF-EPS from 51 members to $5 \mathrm{RMs}$, although it takes into account the cluster population in the post processing stage. The impact of ensemble size reduction needs to be carefully assessed for first and higher order moments. Previous studies with GCMs (Toth and Kalnay, 1997; Leith, 1974; Houtemaker and Derome, 1995) show that only minor improvement in forecast skill is obtained beyond 10 members, but the spatial and temporal relationship between spread and error improves well beyond this limit. This is an open question in LEPS.

Our LEPS horizontal resolution is $12 \mathrm{~km}$ mainly to limit computational requirements for operational purposes. However, the Hi-Res forecast has better objective scores and reveals more detailed and defined mesoscale structures when a subjective analysis is performed. As computer power continues to increase, LEPS with horizontal grid spacing less than $10 \mathrm{~km}$ will become feasible in the near future, and additional work will be required to find the best compromise between performance, LEPS RMs number, and horizontal resolution.

Acknowledgements. This work was realized in the framework of the project "Sviluppo di Distretti Industriali per le Osservazioni della Terra" funded by "Ministero dell'Università e della Ricera Scientifica". We are grateful to APAT - Dipartimento Tutela delle Acque Interne e Marine for raingauge data. We are grateful to the Italian Air Force and ECMWF for MARS database account. We are grateful to the reviewers for their thoughtful suggestions.

Edited by: O. Talagrand

Reviewed by: K. Mylne and another referee

\section{References}

Baldwin, M. E.: QPF verification system documentation, http://www.emc.ncep.noaa.gov/mmb/ylin/pcpverif/scores/docs/ mbdoc/pptmethod.html, 2000. 
Brooks, H. E., Doswell, C. A., and Maddox, R. A.: On the use of mesoscale and cloud-scale model in operational forecasting, Wea. Forecasting, 7, 120-132, 1992.

Colle, B. A., Mass, C. F., and Westrick, K. J.: MM5 precipitation verification over the Pacific Northwest during the 1997-99 cool seasons, Wea. Forecasting, 15, 137-154, 2000.

Cotton, W. R., Pielke, R. A. Sr., Walko, R. L., Liston, G. E., Tremback, C. J., Jiang, H., McAnelly, R. L., Harrington, J. Y., Nicholls, M. E., Carrio, G. G., and McFadden, J. P.: RAMS 2001: Current status and future directions, Meteor. Atm., 82, 529, 2003.

Federico, S., Bellecci, C., and Colacino, M.: Quantitative precipitation of the Soverato flood: the role of orography and surface fluxes. Nuov. Cim. C, 26 C, 7-22, 2003.

Federico, S., Avolio, E., Bellecci, C., Colacino, M., Lavagnini, A., Accadia C., Mariani, S., and Casaioli, M.: Three model intercomparison for quantitative precipitation forecast over Calabria. Nuov. Cim. C, 26 C, 627-647, 2004.

Houtemaker, P. L. and Derome, J.: Methods for ensemble prediction, Mon. Wea. Rev., 123, 2181-2196, 1995.

Houtemaker, P. L., Derome, J., Ritchie, H., and Mitchell, H. L.: A systematic simulation approach to ensemble prediction, Mon. Wea. Rev., 118, 1429-1443, 1996.

Leith, C. E.: Theoretical skill of Monte Carlo forecasts, Mon. Wea. Rev., 102, 409-418, 1974.

Marsigli, C., Montani, A., Nerozzi, F., Paccagnella, T., Tibaldi, S., Molteni, F., and Buizza, R.: A strategy for high-resolution ensemble prediction, Part II: limited-area model experiments in four Alpine flood events, Quart. J. Roy. Meteor. Soc., 127, 20952115, 2001.

Mason, S. J. and Graham, N. E.: Conditional probabilities, relative operating characteristics and relative operating levels, Wea. Forecasting, 14, 713-725, 1999.

Mass, C. F., Ovens, D., Westrick, K., and Colle, B. A.: Does increasing horizontal resolution produce more skilful forecast? Bull. Am. Met. Soc., 83, 407-430, 2002.

Molinari, J. and Corsetti, T.: Incorporation of cloud-scale and mesoscale down-drafts into a cumulus parametrization: results of one and three-dimensional integrations, Mon. Wea. Rev., 113, 485-501, 1985
Molteni, F., Buizza, R., Marsigli, C., Montani, A., Nerozzi, F., and Paccagnella, T.: The ECMWF Ensemble Prediction System: Methodology and validation, Quart. J. Roy. Meteor. Soc., 122, 73-119, 1996.

Molteni, F., Buizza, R., Marsigli, C., Montani, A., Nerozzi, F., and Paccagnella, T.: A strategy for high-resolution ensemble prediction, Part I: definition of representative members and global model experiments, Quart. J. Roy. Meteor. Soc., 127, 20692094, 2001.

Montani, A., Marsigli, C., Nerozzi, F., Paccagnella, T., Tibaldi, S., and Buizza, R.: The Soverato flood in Southern Italy: performance of a global and limited-area ensemble forecasts, Nonlin. Proc. Geophys., 10, 261-274, 2003.

Pielke, R. A., Cotton, W. R., Walko, R. L., Tremback, C. J., Lyons, W. A., Grasso, L. D., Nicholls, M. E., Murran, M. D., Wesley, D. A., Lee, T. H., and Copeland, J. H.: A comprehensive meteorological modelling system-RAMS, Meteorol. Atmos. Phys., 49, 69-91, 1992.

Smith, R. B.: The influence of mountains on the atmosphere, Advances in Geophysics, 21, Academic Press, 87-230, 1979.

Stanski, H. R., Wilson, L. J., and Burrows, W. R.: Survey of common verification methods in meteorology. WMO World Weather Watch Tech. Report, 8, 1989.

Toth, Z. and Kalnay, E.: Ensemble forecasting at NMC: the generation of perturbations, Bull. Am. Met. Soc., 125, 2317-2330, 1994.

Toth, Z. and Kalnay, E.: Ensemble forecasting at NCEP and the breeding method, Mon. Wea. Rev., 125, 3297-3319, 1997.

Walko, R. L., Tremback, C. J., Pielke, R. A., and Cotton, W. R: An interactive nesting algorithm for stretched and variable nesting ratio, J. Appl. Met., 34, 994-999, 1994.

Walko, R. L., Cotton, W. R., Meyers, M. P., and Harrington, J. Y.: New RAMS cloud microphysics parameterization part I: the single-moment scheme, Atmos. Research, 38, 29-62, 1995.

Walko, R. L., Band, L. E., Baron, J., Kittel, T. G., Lammers, R., Lee, T. J., Ojima, D., Pielke, R. A. Sr., Taylor, C., Tague, C., Tremback, C. J., and Vidale, P. L.: Coupled Atmosphere-BiosphereHydrology Models for environmental prediction, J. Appl. Met., 39, 931-944, 2000.

Wilks, D. S.: Statistical methods in the atmospheric sciences, Academic Press, San Diego, CA, USA, 1995. 